\title{
Process Evaluation of the MOSAIC trial, Part I: Therapist Experiences of Delivering Two \\ Psychological Therapies for Treatment of Anorexia Nervosa
}

Daniella Waterman-Collins, $\mathrm{MSc}^{1^{*}}$

Beth Renwick, $\mathrm{BSc}^{1^{*}}$

Anna Lose, MSc ${ }^{1}$

Martha Kenyon, $\mathrm{MSc}^{1}$

Lucy Serpell, PhD, DClinPsy ${ }^{2,5}$

Lorna Richards, FRCPsych ${ }^{3}$

Nicky Boughton, $\mathrm{MA}^{4}$

Janet Treasure, MD PhD FRCP FRCPsych ${ }^{1}$

Ulrike Schmidt, MD PhD FRCPsych ${ }^{1}$ on behalf of the MOSAIC trial group.

\section{*: These authors contributed equally}

1) Section of Eating Disorders, Department of Psychological Medicine, Institute of Psychiatry, King's College London, London

2) Hope Wing, Porters Avenue Health Centre, North East London Foundation Trust, Dagenham, Essex

3) The Phoenix Wing, St Ann's Hospital, Barnett, Enfield and Haringey Mental Health Trust, Tottenham, London

4) Oxford Adult Eating Disorder Service, Cotswold House, Warneford Hospital, Oxford NHS Foundation Trust, Oxford

5) Eating Disorders Research Group, Research Dept. of Clinical, Educational \& Health Psychology, University College London, London

\section{Corresponding Author}

Ulrike Schmidt, PO Box 59, Section of Eating Disorders, Institute of Psychiatry, King's College London, De Crespigny Park, London, SE5 8AF, Ulrike.schmidt@kcl.ac.uk

This article presents independent research commissioned by the National Institute for Health Research (NIHR) under its Programme Grants for Applied Research scheme (RP-PG-0606-1043). The views expressed in this publication are those of the author(s) and not necessarily those of the NHS, the NIHR or the Department of Health. This work was supported by a grant from the NIHR Biomedical Research Centre for Mental Health, South London and Maudsley NHS Foundation Trust and Institute of Psychiatry, King's College London. 


\begin{abstract}
Objectives: Forming part of a process evaluation of a large RCT [The Maudsley Outpatient Study of Treatments for Anorexia Nervosa and Related conditions (MOSAIC)] comparing two outpatient therapies for Anorexia Nervosa: The Maudsley Model for Treatment of Adults with Anorexia Nervosa (MANTRA) and Specialist Supportive Clinical Management (SSCM) this study adopted a qualitative approach to examine therapist experiences of treatment delivery.
\end{abstract}

Method: Twenty MOSAIC therapists completed semi-structured interviews. Interviews were recorded, transcribed and analysed thematically.

Results: Themes of positive aspects, challenges and therapeutic fit emerged. MANTRA was seen as structured and flexible but could feel demanding on therapist time and skill. The slow pace and narrower focus of SSCM gave patients space to talk, but the lack of psychological tools and nutritional emphasis could create frustration. Views on the therapeutic relationship and patienttherapy fit differed across treatments.

Discussion: Findings provide testable hypotheses about what works for whom, ideas for therapist training, treatment development and delivery.

Key words: Anorexia Nervosa, Qualitative Research, Process Evaluation, Randomised Controlled Trial, Patient 


\section{Process Evaluation of the MOSAIC trial, Part 1: Therapist Experiences of Delivering Two Psychological Therapies for Treatment of Anorexia Nervosa}

Anorexia Nervosa (AN) is a serious and often chronic mental disorder, with major impacts on both psychological and physical health. It has high levels of mortality, disability and disease burden (Butterfly Report, 2012). Clinical trials evaluating outpatient treatments for adults with AN are scarce and have had disappointing outcomes (Watson \& Bulik, 2012). Thus far, no high quality empirical evidence exists to support a preferred psychological model (Hay, Touyz, \& Sud, 2012; Hay, Bacaltchuk, Stefano, \& Kashyap, 2009). The National Institute of Clinical Excellence (2004) has highlighted the importance of developing and evaluating novel approaches to effectively treat AN.

Recently, we developed a novel out-patient therapy for adults with AN (Maudsley Model of Anorexia Nervosa Treatment for Adults, MANTRA). This is a modularised, manual-based therapy, developed from a biologically informed, trait-focused maintenance model of AN (Schmidt \& Treasure, 2006; Treasure \& Schmidt, 2013). MANTRA addresses four intra- and inter-personal maintaining factors which are: thinking style (e.g. cognitive inflexibility, detail focus at the expense of the bigger picture), impairments in socio-emotional processing (including avoidance of the experience and expression of emotions), positive beliefs about anorexia and unhelpful responses of close others to the illness (i.e. high expressed emotion, accommodation and enabling of symptoms). The patient manual contains tasks and exercises to complete within and outside of therapy as well as personal letters (e.g. caseformulation, good-bye letter) from the therapist at the middle and end stages, reflecting on progress made within therapy. The MANTRA model and therapy were developed using the revised Medical Research Council (MRC) framework for complex interventions (MRC., 2008). This has involved an iterative translational process between basic research and model/treatment development, combining a top-down (theory-led, data-driven, quantitative) and bottom-up (patient and clinician experience led, qualitative) approach. Manual development was based on the advice of Carroll and Nuro (2002) for treatment development. A case series (Wade, Treasure, \& Schmidt, 2011) and a pilot 
randomised controlled trial (RCT) (Schmidt, et al., 2012) testing MANTRA have shown promising results and the intervention has since been significantly refined. We are now conducting a large scale RCT [the MOSAIC trial - Maudsley Outpatient Study of Treatments for Anorexia Nervosa and Related Conditions] evaluating efficacy of MANTRA against a credible comparison treatment, Specialist Supportive Clinical Management (SSCM; Mclntosh, et al., 2006). With a total of 142 participants this is one of the largest AN treatment trials to be conducted.

SSCM was originally designed to mimic routine outpatient treatment. It combines features of clinical management (Fawcett, Epstein, Fiester, Elkin, \& Autry, 1987), such as education, care, support and building a strong therapeutic relationship with supportive psychotherapy (Dewald, 1994), which involves helping patients through reinforcement, assurance and advice. Its main aim is to restore healthy eating and a normal weight by focusing on patient specific target symptoms. In a previous RCT, SSCM emerged as superior to Cognitive Behavioural Therapy (CBT) and Interpersonal Psychotherapy (IPT) (Mclntosh, et al., 2005).

In the MOSAIC trial, 142 adult outpatients with AN were randomly allocated to receive either MANTRA or SSCM. Therapists completed training in both treatment modalities and patients in either treatment receive 20 to 30 one-hour weekly outpatient sessions. Full details of the study protocol of the MOSAIC trial can be found elsewhere (Schmidt, et al., 2013).

Although RCTs are the 'gold standard' in the evaluation of complex interventions, guidelines from the UK Medical Research Council (2008) and a comprehensive systematic review (Greenhalgh, Robert, Macfarlane, Bate, \& Kyriakidou, 2004) recognise that combining outcome evaluations with understanding of therapy process 'can provide useful insights into why an intervention achieves or fails to achieve the expected outcomes'. They recommend including the perspectives of clinicians, patients and other stakeholders as early as possible in the design, further development and 
dissemination of novel treatments. Other trialists, too, have suggested that such process evaluations are a valuable way of obtaining information, which would not be obtained from quantitative outcome data alone (Crawford, Weaver, Rutter, Sensky, \& Tyrer, 2002; Oakley, Strange, Bonell, Allen, \& Stephenson, 2006). Oakley, et al., (2006) noted that 'process evaluations are especially necessary in multisite trials, where the "same" intervention may be implemented and received in different ways' and that such data should be collected from all participating centres and be analysed before outcome data to avoid bias in interpretation. Commonly, such process evaluations involve qualitative data.

To study therapy process in the MOSAIC trial, we conducted two parallel qualitative studies that explored therapist and patient experiences of MANTRA and SSCM with the idea that combining both clinicians' and patients' perspectives will provide a more in-depth understanding of these two treatments and to inform future intervention development and therapist training. The first part which focuses on the experiences of therapists is reported here.

Exploration of therapists' experiences of treatment delivery has been largely neglected in the eating disorder literature. Only three studies have empirically investigated therapist experiences of delivering therapies for ED (Easter \& Tchanturia, 2011; Jarman, Smith, \& Walsh, 1997; Smart, Tsong, Mejía, Hayashino, \& Braaten, 2011). However, there has been no systematic inquiry into the views and experiences of therapists delivering specific treatments in a large RCT such as MOSAIC. The aims of the present study were to obtain therapists' views on unique and overlapping features of these two treatments, therapists' perceptions about the relative merits of these treatments for different patients, their preferences for and dislikes of certain elements of these therapies, and the challenges they experienced in delivering these two treatments. 


\section{Setting, Recruitment and Participants}

20 of the 29 therapists involved in the MOSAIC trial were recruited from the four centres involved; South London and Maudsley NHS Foundation Trust, North East London Foundation Trust, Barnet, Enfield and Haringey Mental Health Trust and Oxford Health NHS Trust. All therapists received training in both MANTRA and SSCM, with ten having delivered both at the time of interview. Over two thirds of patients had been recruited into the trial at the time of interview, collecting data at this time ensured both treatments were fresh in therapists' minds and allowed interviewers to capture different degrees of therapist experience. Therapists were highly experienced in the treatment of EDs, with the mean number of years in the field being 7.6. (see Table 1 for therapist demographics). Seventeen female and three male therapists were interviewed. One therapist had been involved in developing the MANTRA manuals (participant number: T1). There were no exclusion criteria and reasons for non-participation included annual leave or leaving the service. Ethics approval had already been granted to conduct the MOSAIC trial (Reference: 10/H0714/9). Amendments to the protocol were subsequently approved by the NHS Ethics committee.

\section{Procedures}

\section{Interviews}

Two researchers (DWC; CD) conducted face-to-face individual semi-structured interviews lasting approximately 30 minutes. Therapists provided written informed consent and were assured that all comments were confidential and non-attributable to them as individuals. Anonymity in both transcribing and data analyses was guaranteed. The British Medical Journal's guidelines for the conduct of qualitative research were followed (Kuper, Lingard, \& Levinson, 2008).

The topic guide, which was devised by the research team, had three sections (interview guide is available on request). The first section covered therapist demographic information, the second asked broad, open ended questions about the treatments. Subsequently, the individual stages of 
each therapy, as well as comparisons between the two were made. Therapists were asked about how each therapy impacted on the therapeutic relationship and whether they felt they were able to meet patients' needs. Therapists who had only delivered one therapy were unable to make comparisons, but were asked to discuss their expectations of delivering the other therapy.

\section{Data Analysis}

Coding Process and Thematic Analysis: The semi-structured interviews were transcribed and imported into NVivo (version 10; QSR International). Data were initially analysed by DWC according to the six phase process of thematic analysis, outlined by (Braun \& Clarke, 2006). This method allows the researcher to gain insight into respondents' subjective account of their experiences by examining the data unit by unit. Firstly, the data were 'cleaned' and transcripts re-read, with key ideas being noted down. All data were then independently and systematically coded, where a code refers to the most basic segment or element of the raw data and identifies a feature of the data that appears interesting to the analyst. Initially as many codes as possible are generated and at this stage all speculative accounts given by therapists who had only delivered one therapy were excluded from analysis. All other data extracts were collated together within each individual code. The long list of codes were sorted and combined into potential over-arching themes, with others forming subthemes within themes. The themes were then reviewed and edited to further break down large and complex themes or to discard those which have too little supporting data. Broad, high-order themes and subthemes were labelled and organised to include all relevant codes. Data triangulations were completed in order to improve validity and reliability of the resulting themes. This involved BR and US independently coding the data, noting down over-arching themes and additional ideas. When disagreement occurred the three researchers consulted until a consensus was reached. The final thematic map was then created (figure 1).

\section{RESULTS}


The resulting themes were separated into sections of MANTRA and SSCM. Both sections consisted of 3 super-ordinate themes with a number of sub-themes in each. Figure 1 gives a diagrammatic overview of the distribution of these super-ordinate themes and sub-themes for both MANTRA and SSCM

\section{MANTRA}

\section{POSITIVES AND HELPFUL ASPECTS}

Structure of Therapy and Manual: 79\% (11/14) of therapists spoke about MANTRA being wellstructured with clear, yet fluid treatment phases and that this was helpful to both them and the patient:

T14: 'Manual is brilliant, I think it gives a very good framework and I love it.'

T11: 'I like having a book which they can have it all in, doing exercises, the structure of it, it's excellent really.'

13/14 (93\%) Therapists also commented that the way the topics and material in the treatment/manual were sequenced made sense to them.

T2: 'it makes sense, it moves logically'.

T11: 'It's a really nice structure, so it's just, it's essentially, motivation, a bit on nutrition, formulation, and then kind of the main part of the therapy whatever it is you're going to focus on at the end.'

Flexibility/Tailoring of Therapy: $93 \%(13 / 14)$ of therapists felt that within the overarching structure the treatment had the flexibility for them to collaboratively choose sections from the manual to tailor the treatment to the patient's illness stage and needs.

T20: 'Focus on what's discussed is very much negotiated with the client, which I really like and really agree with'. 
T11: 'I think it's got everything in there that you'd need. It's very detailed, it's been excellent...you really can tailor it, more than any other therapy, because there's just so much'.

Manual as a Tool: $14 / 14(100 \%)$ All therapists mentioned being able to use the manual as a 'backbone' and tool to guide therapy.

T15: 'I took it as very much as guidance, I think that's really important, that the patient can scribble along and write whatever they want, it's not owned by us...'

T9: 'Even if you're less experienced, it gives a really good basis for someone to learn what's important and relevant when treating someone with AN...I see it more as a resource now, based on the formulation and what we do at the beginning. It's not about covering all exercises.'

9/14 (65\%) Therapists also mentioned different parts of the manual that they thought had been helpful:

T4: [talking about the formulation] 'It's quite a nice bit actually, really and its quite empowering for them to see the petals come together, and say, oh, okay this is me and I can really see all these different factors influence, that's quite nice, that's quite good.'

T2:'... I think all of the emotional/social mind stuff is absolutely fabulous'

T11: 'the bit I like the most is the thinking style module, because in CBT although you do kind of, ... you know patients think in very inflexible and in detailed ways, but you don't really tackle it at all I don't think...'

Additionally, therapists liked that patients could keep the manual and use it for reference and something to draw upon on after treatment: 
T5: 'Give it to them to keep working on, once you've got them on the right path...at the end they still have a massive toolkit to use for as long as they need'.

T9: 'In the longer term, they've got more skills and they've got the workbook that they can keep with them'.

\section{CHALLENGES}

Demands on Therapist Skill: 14/14 All therapists noted the large amount of material and choice within the manual and that this had the potential for making the therapy feel busy, demanding or even overwhelming for therapist and patient.

T2: 'I would probably say that MANTRA is more all encompassing but harder work both I think for therapist and for patient because it involves more'

T11: 'at first it felt overwhelming because it was this huge manual and l've never done anything manual based before, ..., but now that I kind of got used to it and I know kind of what's coming next and the structure of it, it doesn't feel quite so intimidating and... it's excellent really.'

$10 / 14(71 \%)$ Therapists also felt that care needed to be taken as to how the manual was introduced to patients at the beginning of therapy:

T1: '[patients] may feel overwhelmed by it, you have to be very careful with how you introduce it, and don't put people off...early phase in MANTRA, the danger is to talk too much about what's in the manual.'

$6 / 14$ (42\%) Some therapists found the formulation section hard, although this became easier as they became more familiar with the manual:

T6: 'Doing the formulation is quite hard work; especially all those petals are quite confusing. I think it should have been simpler to get your head round them, it was quite hard and muddling, that's quite difficult.' 
T11: 'I didn't like the formulation, I thought it was too much, it considers all the different areas that could be maintaining anorexia, but I think that was lack of experience, because now l've done it a few times I do really like it.'

4/14 (29\%) Other therapists mentioned that given the fixed number of sessions offered within the MOSAIC trial, the middle section of treatment could feel rushed:

T5: 'Middle...working more towards active changes, can be a bit tricky because sometimes you've got lots of factors that you've identified as being in the formulation... we've only got 10 sessions to try and work through all these things. It can feel a bit squashed.'

Time Demands on Therapists: 4/14 (29\%) of therapists mentioned that having to write personal formulation and good bye letters to patients made extra demands on their time, although all acknowledged that the letters were a valuable tool and that patients liked them very much.

T1: 'You have to write a formulation letter, and you have to write an end of therapy letter, and it's always a bit irritating to find room for writing these letters, but once you've done it you actually know your patient much better because it means you really force yourself to reflect much more on a particular patient.'

\section{THERAPEUTIC FIT}

Therapeutic Relationship: 9/14 64\% of therapists described how the therapeutic stance of motivational interviewing was thought to be key in helping build a positive therapeutic relationship:

T20: 'The person-centeredness, flexibility, always asking if it's okay and what to do, working things out together about how to do it, I find has really helped strengthen the relationship'

T12: 'You have to cover them with warm empathic goo, and I think the first handful of sessions with MANTRA, that's what you do'. 
The majority of therapists $8 / 14(57 \%)$ also thought that the module addressing the valued nature of AN, the beginning of the manual, was helpful in engaging patients:

T4: 'Motivational aspect at the beginning is a good way of getting that therapeutic alliance'.

T7: 'Shared understanding....idea that you're working together in trying to understand what's going on'.

5/14 (37\%) However, therapists were also aware that working with the manual could get in the way of building a therapeutic relationship. Some patients might feel they were forced down a particular program, with therapeutic tasks potentially perceived as mindless activities that hindered the therapeutic bond:

T5: 'Some people find that quite off-putting, that it's not individual or flexible enough...might make people feel a bit pigeon holed....just filling in boxes and paper rather than actually connecting. I think it's about how the manual is used to help us along the way'.

T1: 'They may feel overwhelmed by it as well, so you have to be very careful with how you introduce that, and that you don't, sort of put people off... may feel patronised by it, some people will think it looks too girly, too young, the men might not like it... we have people who are often a bit, rigid, and a bit black and white in their thinking, so they either take to it or they will violently dislike it.'

QT04: 'really difficult I think when there's a lot going on with our patients to try and bring it back and not address it and bring it back to the structure of the programme. That was difficult because it then affected my ability to communicate with her and support her in that way.'

Subsequently, the individualised diagrammatic formulation was deemed important, alongside the use of formulation and goodbye-letters:

T9: 'I really like how that's laid down [formulation], and how we share it with the patient, in the form of the letter.'

T5: 'The formulation letter is something that is quite good for the therapy relationship and I think the goodbye letter is a really nice tool to help with the ending of reflecting on the therapy and saying goodbye to each other and I think that's quite nice'. 
What Works for Whom: $8 / 14(57 \%)$ therapists thought that MANTRA might be suitable for a wide range of patients, ranging from the more 'typical' cases of restricting AN with rigid and perfectionist traits, to those with more complex presentations:

T18: 'Clients who are quite to the point, structured, perfectionist, then MANTRA would possibly match them better in a way, a more systematic approach, covering different areas, and quite planned'

T7: 'Can cover a broader range of patients really, I think it's more accessible... whatever's going on for each of them, it feels like MANTRA can cover that.'

Additionally, 4/14 (29\%) thought that MANTRA may suit AN patients who lacked insight into their illness and were ambivalent about treatment:

QT03: 'Who like a modular, manualised approach, who like to have concrete things to be working on, and to have something tangible in their hand.... and also that it just moves quite gently through'. QT01 MANTRA has a lot of tools for tackling the eating disorder in a roundabout way, and coming back to the eating disorder issues, whilst talking about some other relevant things that might be contributing to the eating disorder, so you build up your relationship, and you teach people things that might be helpful in chipping away at their reluctance and often lack of insight to change'

T5: 'If someone was much more ambivalent in terms of their motivation, if they didn't really have a good sense of what was going on with their eating problems and why it was happening, what was making them stuck, I'd probably use a MANTRA approach'.

8/14 (57\%) of Therapists suggested MANTRA might work less well for patients who were dyslexic, male or didn't respond well to structure and homework, and some thought it would work less well for individuals with complex co-morbidities (but see T5 above):

T16: 'If find reading difficult, or they're dyslexic and find writing hard work, it might be just that bit 
too much for them....older patients are not so keen, it feels like homework'.

T5: 'more atypical or binge/purge anorexic or someone who has a more chaotic presentation, it can be a bit hard; there is nothing in the manual about self-harm for example, or alcohol, drugs, bingeing, and other co-morbidities'.

\section{$\underline{\text { SSCM }}$}

\section{POSITIVES AND HELPFUL ASPECTS}

Space to Talk: 53\% (8/15) of therapists delivering SSCM highlighted the positive effect of allowing patients to bring and discuss what they wanted to each session:

T10: 'There's a lot of room allowing you to take it off to meet the needs of the client'.

T5: 'getting to know the patients, it's a very slow gentle model'

$12 / 15(80 \%)$ Therapists spoke about a less defined structure or set agenda, allowing patients the space to talk and feel re-assured, whilst receiving expert advice. Some patients found this helpful and therapists were often surprised at how far this technique could go with some individuals:

T7: 'It's not very busy so I think there is an aspect on just focusing on the two of you in the room, it's a space to allow things to develop between you and the patient... showing empathy and interest can make a difference'.

T1: 'what it showed me was that, you can go a very long way with...being quite slow, doing quite simple things with people, listening to them a lot...trying not to do too much in a session'

This included having time to get patients to think about their nutrition and how to change this.

T13: 'You're very explicitly giving people handouts [on nutrition], you're devoting a big chunk of the first 5 sessions to that, in fact all three people have really liked it and have just said, that made a real impact...you know giving due emphasis to that, and also I think it's sort of takes away 
this awful self-blame that people have I think in seeing people with this group of patients who are so you know, blame themselves...,'

Set own Goals: $8 / 14(53 \%)$ of therapists highlighted the importance of allowing the patients to set their own goals, based upon their target symptoms. Patients may have found this beneficial as it allowed them to focus on more specific needs which were personal to them.

T17: 'Can spend the whole session talking about what they wanted to talk about and I think for some, that's really good...flexibility to bring what they want, and set their own goals'.

T2: "I enjoyed the beginning, I think that the development of the rapport, the explaining what needs to be done, the setting of goals in terms of weight, I think felt very comfortable for me, felt comprehensive.'

\section{CHALLENGES}

Focus on Eating and Weight Restoration: 73\% (11/15) of SSCM therapists mentioned how the central focus of weight restoration and target symptoms at times felt frustrating and somewhat limiting. This also applied to the standardised SSCM formulation:

T9: 'Focus on eating, I find sometimes that can feel quite tedious, when you'd like to be focusing on other thing [and the] formulation, I feel was less useful and comprehensive and it doesn't seem to guide treatment as much, I suppose there is a worry that you feel quite constrained and a lot of things you couldn't do....didn't feel very much of a toolkit or things I could use for them'.

T8: 'I think the middle is all about, it's about 12-14 sessions, can feel quite long as there's not actually very much change, and unless you've got a good momentum and you're working on things, you're actually doing the same thing each week, so it's quite repetitive'. 
Lack of Therapeutic Tools: $73 \%(11 / 15)$ of therapists described how SSCM did not allow them to address patients' psychological difficulties in any depth. There was a sense of frustration amongst most therapists:

T17: 'feels like my hands are tied a bit as I can't just be myself and ask them about something and go into things in a lot more detail like I normally would'.

This was particularly apparent in situations where patients presented with more complex problems or co morbidities, where SSCM does not provide the tools to address these:

T1: 'Frustrating that when you identify a particular difficulty or clinical problem in people... and you think they could benefit from this specific approach, then you couldn't actually do it'.

T7: 'I think it's quite limiting really, if there's any other sort of complications.... it was difficult; if they needed more, I felt restricted by it'.

Inflexibility/Lack of Tailoring: $60 \%(9 / 15)$ of therapists found working with an atypical presentation or lack of motivation to change a challenge. Being unable to tailor treatment to patients meant sessions could really drag on, become repetitive or would sometimes finish early, adding to the frustration:

T8: 'I don't find the model of SSCM very helpful at all and actually quite hard to tailor. It's very different from MANTRA or CBT, which have an individualised formulation'.

T10: 'It feels a bit disabling, seeing that 20 therapy minutes, that's pretty much it. It feels we are wasting session time; we can do more if I could use the other tools'.

\section{THERAPEUTIC FIT}

Therapeutic Relationship: 73\% (11/15) of therapists suggested that the slower pace of SSCM and the relative lack of a fixed agenda, except from focusing on weight restoration and target symptoms, was helpful in developing rapport and building up trust: 
T9: 'You've got time to develop a therapeutic relationship and you're there listening and being supportive; you can move people forward in that way'.

T7: 'not very busy so I think that it can mean there is an aspect of just focusing on the two of you in the room and it's a space to allow things to develop between you and the patient....and really just showing empathy, showing interest in them, that in itself can make a difference.'

$12 / 15(80 \%)$ of Therapists also highlighted things that may get in the way of building a relationship. For example, the lack of specific techniques within SSCM for addressing patients' more complex problems and psychological needs may leave the patient feeling frustrated in their ability to tackle the root of their problem and see real change:

T8: 'The patient maybe feels a bit frustrated if they've got ideas and strategies for change, it feels a bit like the therapist is expecting it all to come from them'.

T2: 'I felt slightly lost; I couldn't quite find a way of helping her to challenge this [low self esteem and social anxiety] in the model. And although there was lots of empathy and encouragement, perhaps a little more specific work could have helped her along and made her feel that it was more worthwhile'.

What Works for Whom? 60\% (9/15) of therapists felt that SSCM worked for some patients, but was suitable for a more limited range of individuals. Therapists suggested that SSCM might work best for those with less complex presentations, with high motivation to change and a willingness to address target symptoms:

T10: 'Talkative, more engaging, more motivated, willing to understand and comprehend what's happening, share their own experiences; someone like that may benefit more from SSCM.

T18: 'Looking for a bit of support, somebody who listens and who understands, then I think that would be SSCM, definitely'. 
T7: 'especially the medical side of it, really helps them think about that a lot, identifying the target symptoms, weighing, focusing on that, it causes them to think about that quite a lot.

13/15 (87\%) Therapists suggested SSCM might work less well if patients were unwilling to directly confront the issue of food and weight restoration and were in denial about their target symptoms, needed something more structured, had more complex psychological issues or were not very talkative:

T7: 'SSCM is quite limiting, it just feels like it's for a narrower range of patients that it works for.'

T17: 'Have people that really aren't responsive... in SSCM it's like what are the activities? You've got some handouts, you've got the 'formulation' sheet, but other than that, you can easily just sit there being like, "what do you want to talk about today?"'

5/15 (33\%) Therapists proposed SSCM as the first stage of therapy in a stepped care approach, as it may be sufficient for some individuals:

T13: 'I think that there's a proportion of patients we see that it could really help...lf it works you could transfer it into primary and secondary care, which would mean that people can have SSCM and if it doesn't work, they can be referred to specialist services. You can try and clear out some people who actually could have been helped.'

\section{DISCUSSION}

This study is the first part of a process evaluation conducted in the context of the MOSAIC trial. The second part concerning the experiences of patients is reported separately (Lose, et al., Submitted). The present study provides insight into how a diverse group of therapists who delivered MANTRA and/or SSCM to patients with AN in the context of a large RCT, perceived these very different treatments. Distinct themes emerged for MANTRA and SSCM in relation to positive aspects, challenges and therapeutic fit. MANTRA was seen as a structured, yet flexible approach that centred 
on a manual. This was perceived as a valuable therapeutic tool and allowed individual tailoring of therapy. However, MANTRA was also felt to be more demanding on therapists in terms of needing to be very familiar with the content and model to utilise it optimally. The need to spend more time preparing for sessions, in order to sequence and pace therapy content and process appropriately, and being mindful of not overloading the patient was also highlighted. In contrast, SSCM was felt to be less demanding on therapists as there was less material to cover. Allowing patients' space to talk was a positive, but SSCM was felt to be somewhat limited, given its strong focus on food and eating, and lack of other therapeutic procedures and tools.

Therapists felt that both therapies allowed them to develop a positive therapeutic relationship. The explicit motivational interviewing stance combined with shoulder-to-shoulder therapeutic exercises and the use of letters from the therapist to the patient were useful in MANTRA. In SSCM the largely supportive stance and time to listen and attend to patients were remarked upon. Therapists perceived these treatments as clearly very different, with significant and unique strengths and some frustrations or concerns. As such this indicates that therapists had a solid understanding of what these treatments are about, were trying to implement them with fidelity and found both of them largely acceptable.

Furthermore, therapists gave their views on what kinds of patients might be more suited to MANTRA or SSCM. There seemed to be consensus that MANTRA would work for a wider range of patients, including those with 'classical' restricting AN, but also those with poor motivation and more complex and idiosyncratic problems. However, there was some difference of opinion as to how well it fitted patients with more bulimic and chaotic presentations. In contrast, SSCM was thought to be suited to patients with milder forms of the illness, those who were highly motivated to work on improving their eating and those with previous experience of more structured therapies. 
Whilst therapists may find using certain therapeutic techniques and procedures more enjoyable and rewarding, and hold certain beliefs about what appears to work best within treatment, this may not necessarily coincide with what are the most beneficial and effective interventions as measured by improvements in ED symptoms and also from the individual patient's point of view. For example, in the present study therapists often reported feeling frustrated by the strong focus on normalising eating and weight in SSCM and the lack of other therapeutic manoeuvres. Yet in one previous trial SSCM was superior to CBT and IPT (Mclntosh, et al., 2005) and in another it was overall as effective as MANTRA (Schmidt, et al., 2012). Moreover, we know that many AN patients value simplicity and predictability (Vitousek \& Ewald, 1993) and SSCM may to some extent embody that. Having said that, one previous quantitative study in ED found remarkable agreement between patients and therapists with regard to their views on effective aspects of treatment (Vanderlinden, Buis, Pieters, \& Probst, 2007). Likewise, a survey focusing on what constitutes high quality eating disorder services, found remarkable agreement between different stakeholders (patients, carers and clinicians) (Nishizono-Maher, et al., 2011). The overlap between patient and therapist experiences of MANTRA and SSCM will be discussed in the parallel patient experiences paper (Lose, et al., Submitted).

Nonetheless, these findings have some important implications: Firstly, they help generate testable hypotheses in terms of how well MANTRA and SSCM are suited to different patients and what might work best for whom. The MOSAIC RCT is large enough to conduct moderator analyses to assess for example whether MANTRA does indeed have advantages for more complex patients.

Secondly, the findings provide valuable ideas for further treatment development. This is especially important for MANTRA which is an evolving novel therapy. Consideration will need to be given to simplifying and abbreviating materials and/or to provide more in-depth guidance to therapists on which elements of treatment/manual are essential and which are optional. From quantitative 
information about therapy sessions collected in the MOSAIC trial, we will also be able to identify which sections of the manual were used across all cases and which were used more rarely and thus might need to be flagged as optional.

Thirdly, the findings have implications for therapist training. Certainly, training in MANTRA needs to ensure that therapists are highly familiar with the manual and are competent at utilising it appropriately. This is common to all manual-based treatment approaches. Particular attention needs to be paid in training to issues such as how the manual is introduced to the patient, how to combine and sequence the intervention and how a motivational stance is applied to a manual-based approach. The central role of the manual in treatment was felt to potentially be useful for novice therapists who would be able to learn much of what there was to know about treating AN from the manual.

Implications for training in SSCM are less clear. Therapists commented on its simplicity, but therapists in the present study were experienced therapists and it may be that if simple content is delivered without much sensitivity or specialist expertise and skill then it may be less effective.

Fourth, the findings have implications for treatment delivery and dissemination. MANTRA was felt to be 'busy' and could feel 'rushed' whereas SSCM was felt to be slow and unrushed at best, but could feel dragging, repetitive and somewhat limited at worst. In the present study the number of sessions delivered to patients was identical in both treatments, i.e. 20 weekly sessions for patients with a BMI between 18.5 and 15 and 30 weekly sessions for those below BMI 15. In usual clinical practice, session numbers can be adapted to patient needs and interventions can be sequenced. The present findings therefore need to be considered within the constraints of the MOSAIC trial. Optimal delivery of MANTRA may call for a longer treatment duration, e.g. 30 to 40 weekly sessions. Other recent AN studies/trials have used this longer treatment duration (Fairburn, et al., 2013; Wild, et al., 
2009). In contrast, in SSCM if treatment starts 'dragging and getting repetitive' but the patient is not improving, it may be necessary to switch to a different approach.

This study has a number of strengths. To the best of our knowledge it is the first process evaluation conducted within the context of a large AN treatment trial. It is also the first qualitative study to formally capture ED therapists' views on delivering psychological treatments in such a trial. The current study has good ecological validity, using primary data to explore experiences in a naturalistic outpatient setting. A large proportion of study therapists were interviewed across all four centres. The findings reflect a diverse mix of clinicians' views from different therapeutic orientations and provide a rich and comprehensive exploration of experiences. Moreover data were obtained and analysed before the RCT outcome data were known, so as to avoid bias (Oakley, et al., 2006).

Several limitations should also be highlighted. Some therapists had only conducted one of the two therapies, so could not compare them. Moreover, some therapists had only seen a few patients or were yet to complete a whole course of treatment. There is a possibility these factors may have biased opinions. However, therapists are unable to choose which treatment they conduct with each new patient and may never complete a course of treatment due to patient drop out. We therefore view including therapist views from across the treatment timeline a strength of this study as data was collected whilst experiences were fresh in therapists' minds. Additionally, treatments were delivered in the context of a clinical trial with fixed numbers of sessions and other constraints that would not occur in clinical practice. Some of the perceived challenges of delivering these treatments may relate more to the trial context than to the treatments per se.

The findings from the present process evaluation study need to be integrated with those from a sister paper investigating patient experiences (Lose, et al., Submitted). This will produce indication 
as to whether therapist and patient experiences are consistent with each other. Ultimately, both these studies will need to be put in the context of the RCT outcomes.

\section{Acknowledgements}

We thank all the MOSAIC therapists who took part in this study. This article presents independent research commissioned by the National Institute for Health Research (NIHR) under its Programme Grants for Applied Research scheme (RP-PG-0606-1043). The views expressed in this publication are those of the author(s) and not necessarily those of the NHS, the NIHR or the Department of Health. This work was supported by a grant from the NIHR Biomedical Research Centre for Mental Health, South London and Maudsley NHS Foundation Trust and Institute of Psychiatry, King's College London. 


\section{Conflict of Interest}

JT and US are developers of MANTRA, we have no other conflict of interest. 


\section{$\underline{\text { References }}$}

Braun, V., \& Clarke, V. (2006). Using thematic analysis in psychology. Qualitative Research in Psychology, 3(2), 77-101.

Butterfly Report. (2012). Paying the Price: The economic and social impact of eating disorders in Australia. Butterfly Foundation for Eating Disorders, 1-136.

Carroll, K. M., \& Nuro, K. F. (2002). One size cannot fit all: A stage model for psychotherapy manual development. Clinical Psychology: Science and Practice, 9(4), 396-406.

Crawford, M. J., Weaver, T., Rutter, D., Sensky, T., \& Tyrer, P. (2002). Evaluating new treatments in psychiatry: the potential value of combining qualitative and quantitative research methods. International Review of Psychiatry, 14(1), 6-11.

Dewald, P. A. (1994). Principles of Supportive Psychotherapy. American Journal of Psychotherapy, 48, 505-518. 
Easter, A., \& Tchanturia, K. (2011). Therapists' experiences of cognitive remediation therapy for anorexia nervosa: implications for working with adolescents. Clin Child Psychol Psychiatry, 16(2), 233-246.

Fairburn, C. G., Cooper, Z., Doll, H. A., O'Connor, M. E., Palmer, R. L., \& Dalle Grave, R. (2013). Enhanced cognitive behaviour therapy for adults with anorexia nervosa: A UK-Italy study. Behaviour Research and Therapy, 51(1), R2-8.

Fawcett, J., Epstein, P., Fiester, S., Elkin, I., \& Autry, J. (1987). Clinical management-imipramine/placebo administration manual. NIMH Treatment of Depression Collaborative Research Program. Psychopharmacology Bulletin, 23(2), 309.

Greenhalgh, T., Robert, G., Macfarlane, F., Bate, P., \& Kyriakidou, O. (2004). Diffusion of innovations in service organizations: systematic review and recommendations. Milbank Quarterly, 82(4), 581-629.

Hay, P. J., Touyz, S., \& Sud, R. (2012). Treatment for severe and enduring anorexia nervosa: A review. Australian and New Zealand Journal of Psychiatry, 46(12), 1136-1144.

Hay, P. P., Bacaltchuk, J., Stefano, S., \& Kashyap, P. (2009). Psychological treatments for bulimia nervosa and binging. Cochrane Database Syst Rev(4), CD000562.

Jarman, M., Smith, J. A., \& Walsh, S. (1997). the psychological battle for control: a qualitative study of healthcare professionals' understandings of the treatment of anorexia nervosa. Journal of Community \& Applied Social Psychology, 7(2), 137-152.

Kuper, A., Lingard, L., \& Levinson, W. (2008). Critically appraising qualitative research. BMJ, 337.

Lose, A., Davies, C., Renwick, B., Kenyon, M., Treasure, J., \& Schmidt, U. (Submitted). Process Evaluation of the MOSAIC trial. Part II: Patient Experiences of Two Psychological Therapies for Treatment of Anorexia Nervosa. Eur Eat Disord Rev.

McIntosh, V. V., Jordan, J., Carter, F. A., Luty, S. E., McKenzie, J. M., Bulik, C. M., et al. (2005). Three psychotherapies for anorexia nervosa: a randomized, controlled trial. American Journal of Psychiatry, 162(4), 741-747. 
McIntosh, V. V., Jordan, J., Luty, S. E., Carter, F. A., McKenzie, J. M., Bulik, C. M., et al. (2006). Specialist supportive clinical management for anorexia nervosa. International Journal of Eating Disorders, 39(8), 625-632.

Medical Research Council. (2008). A framework for development and evaluation of RCTs for complex interventions to improve health. London: MRC.

National Institute for Clinical Excellence. (2004). Core interventions for the treatment and management of anorexia nervosa, bulimia nervosa and related eating disorders. NICE Clinical Guideline No.9.

Nishizono-Maher, A., Escobar-Koch, T., Ringwood, S., Banker, J., van Furth, E., \& Schmidt, U. (2011). What are the top five essential features of a high quality eating disorder service? A comparison of the views of US and UK eating disorder sufferers, carers and health professionals. European Eating Disorders Review, 19(5), 411-416.

Oakley, A., Strange, V., Bonell, C., Allen, E., \& Stephenson, J. (2006). Health services research: process evaluation in randomised controlled trials of complex interventions. BMJ: British Medical Journal, 332(7538), 413.

Schmidt, Oldershaw, A., Jichi, F., Sternheim, L., Startup, H., McIntosh, V., et al. (2012). Out-patient psychological therapies for adults with anorexia nervosa: randomised controlled trial. $\mathrm{Br} J$ Psychiatry.

Schmidt, \& Treasure, J. (2006). Anorexia nervosa: valued and visible. A cognitive-interpersonal maintenance model and its implications for research and practice. British Journal of Clinical Psychology, 45(Pt 3), 343-366.

Schmidt, U., Renwick, B., Lose, A., Kenyon, M., Dejong, H., Broadbent, H., et al. (2013). The MOSAIC study - comparison of the Maudsley Model of Treatment for Adults with Anorexia Nervosa (MANTRA) with Specialist Supportive Clinical Management (SSCM) in outpatients with anorexia nervosa or eating disorder not otherwise specified, anorexia nervosa type: study protocol for a randomized controlled trial. Trials, 14, 160. 
Smart, R., Tsong, Y., Mejía, O. L., Hayashino, D., \& Braaten, M. E. T. (2011). Therapists' Experiences Treating Asian American Women With Eating Disorders. Professional Psychology-Research and Practice, 42(4), 308.

Treasure, J., \& Schmidt, U. (2013). The Cognitive-Interpersonal Maintenance Model of Anorexia Nervosa Revisited: A summary of the evidence for cognitive, socio-emotional and interpersonal predisposing and perpetuating factors. J Eat Disord.

Vanderlinden, J., Buis, H., Pieters, G., \& Probst, M. (2007). Which elements in the treatment of eating disorders are necessary 'ingredients' in the recovery process? - A comparison between the patient's and therapist's view. European Eating Disorders Review, 15(5), 357-365.

Vitousek, K. B., \& Ewald, L. S. (1993). Self-representation in eating disorders: A cognitive perspective. The self in emotional distress: Cognitive and psychodynamic perspectives, 221-257.

Wade, T. D., Treasure, J., \& Schmidt, U. (2011). A case series evaluation of the Maudsley Model for treatment of adults with anorexia nervosa. Eur Eat Disord Rev.

Watson, H., \& Bulik, C. (2012). Update on the treatment of anorexia nervosa: review of clinical trials, practice guidelines and emerging interventions. Psychological Medicine, 1-24.

Wild, B., Friederich, H. C., Gross, G., Teufel, M., Herzog, W., Giel, K. E., et al. (2009). The ANTOP study: focal psychodynamic psychotherapy, cognitive-behavioural therapy, and treatmentas-usual in outpatients with anorexia nervosa--a randomized controlled trial. Trials, 10, 23. 


\begin{tabular}{|c|c|c|c|c|c|c|c|c|}
\hline \multirow{3}{*}{ Therapist } & \multirow{3}{*}{ Profession } & \multirow{3}{*}{$\begin{array}{l}\text { Yrs in } \\
\text { mental } \\
\text { health }\end{array}$} & \multirow{3}{*}{$\begin{array}{c}\text { Yrs in Eating } \\
\text { Disorders }\end{array}$} & \multirow{3}{*}{$\begin{array}{l}\text { Therapeutic } \\
\text { Orientation }\end{array}$} & \multirow{3}{*}{$\begin{array}{l}\text { Patients } \\
\text { Seen in } \\
\text { MOSAIC }\end{array}$} & \multirow{3}{*}{$\begin{array}{c}\text { Total } \\
\text { MANTRA }\end{array}$} & \multirow{3}{*}{$\begin{array}{l}\text { Total } \\
\text { SSCM }\end{array}$} & \multirow{3}{*}{$\begin{array}{c}\text { Delivered } \\
\text { Both? }\end{array}$} \\
\hline & & & & & & & & \\
\hline & & & & & & & & \\
\hline $\mathrm{T} 1$ & Psychiatrist & 29 & 18 & CBT & 12 & 5 & 6 & Yes \\
\hline T3 & Psychotherapist & 19 & 9 & Integrated & 1 & 1 & 0 & No \\
\hline T4 & Mental Health Nurse & 7 & 4 & Person-Centred & 3 & 2 & 1 & Yes \\
\hline T7 & Psychological Therapist & 13 & 6 & CBT & 6 & 3 & 3 & Yes \\
\hline T8 & Clinical Psychologist & 13 & 11 & CBT & 3 & 0 & 3 & No \\
\hline T9 & Psychologist & 10 & 10 & Integrated & 5 & 4 & 1 & Yes \\
\hline T10 & Psychologist & 7 & 4 & CBT & 1 & 0 & 1 & No \\
\hline $\mathrm{T} 11$ & Clinical Psychologist & 7 & 4 & CBT & 3 & 3 & 0 & No \\
\hline $\mathrm{T} 12$ & Clinical Nurse Therapist & 15 & 13 & Motivational & 5 & 5 & 0 & No \\
\hline
\end{tabular}




\begin{tabular}{|c|c|c|c|c|c|c|c|c|}
\hline T14 & Liaison Nurse & 10 & 8 & Patient-Centred & 2 & 1 & 1 & Yes \\
\hline T15 & Liaison Nurse & 25 & 10 & Integrative & 4 & 2 & 2 & Yes \\
\hline T16 & Psychotherapist & 27 & 14 & CAT & 9 & 7 & 1 & Yes \\
\hline T17 & Clinical Psychologist & 15 & 8 & CBT & 3 & 0 & 3 & No \\
\hline T19 & CBT Therapist & 21 & 4 & CBT & 1 & 0 & 1 & No \\
\hline T20 & Clinical Psychologist & 6 & 3 & CBT & 1 & 1 & 0 & No \\
\hline
\end{tabular}

Table 1: Therapist demographic and MOSAIC trial information

Notes: MOSAIC, The Maudsley Outpatient Study of Treatments for Anorexia Nervosa and Related conditions, MANTRA, The

Maudsley Model for Treatment of Adults with Anorexia Nervosa, SSCM, Specialist Supportive Clinical Management, CBT,

Cognitive behavioural therapy, CAT, Cognitive analytical therapy 